OCU-PHYS-471/AP-GR-142

\title{
Cohomogeneity-one-string integrability of spacetimes
}

\author{
Yoshiyuki Morisawa,, , $*$ Soichi Hasegawa, ${ }^{2}$ Tatsuhiko Koike, ${ }^{3, \oplus}$ and Hideki Ishihara ${ }^{2, \text { 团 }}$ \\ ${ }^{1}$ Advanced Mathematical Institute, Osaka City University, Osaka 558-8585 Japan \\ ${ }^{2}$ Department of Mathematics and Physics, Graduate School of Science, \\ Osaka City University, Osaka 558-8585 Japan \\ ${ }^{3}$ Department of Physics and REC for NS, Keio University, Yokohama 223-8522 Japan
}

(Dated: September 22, 2017)

\begin{abstract}
We present a framework for reducing all possible cohomogeneity-one strings, i.e., strings with geometrical symmetry, in a given spacetime to mechanical systems, and for analyzing integrability of the systems. As applications, it is clarified whether the systems of cohomogeneity-one strings in $\mathrm{AdS}_{5}, \mathrm{AdS}_{5} \times S^{5}$, and $\mathrm{AdS}_{5} \times T^{p, q}$ are integrable or not. This method may reveal a different type of hidden symmetry of spacetimes.
\end{abstract}

\footnotetext{
* morisawa@sci.osaka-cu.ac.jp

† koike@phys.keio.ac.jp

‡ ishihara@sci.osaka-cu.ac.jp
} 
Introduction.- Dynamics of extended object such as string draws much attention in fundamental physics, condensed matter physics, and cosmology [1]. Classical solutions of string dynamics have been widely studied. A variety of stationary solutions and time evolution of strings are investigated [2 $[-6]$ in spacetimes such as flat, de Sitter, anti de Sitter, and black hole spacetimes.

One can treat these string solutions in a unified manner [7-9] because their world sheets share certain symmetry with the spacetimes. Such strings are called cohomogeneity-one (C1) strings and specified by the symmetry (Killing vector field) shared with the spacetime. In that class, the string equation of motion, which is a partial differential equation, is reduced to a system of ordinary differential equations. Namely, motion of the string reduces to motion of a free particle on a projected spacetime, called the orbit space, endowed with a certain weighted metric. Classification of all types of $\mathrm{C} 1$ strings has been carried out in four-dimensional Minkowski spacetime [7] and in five-dimensional anti-de Sitter spacetime [8]. It has also been shown in the former that all types of $\mathrm{C} 1$ strings are integrable, i.e., solved up to quadrature [9].

In this Letter, we develop a general framework of probing spacetime symmetry by C1 strings. It is well-known that motion of a free particle follows a geodesic in the spacetime and integrability of the motion reflects the symmetry of the spacetime. In four-dimensional Schwarzschild spacetime, the isometries thereon immediately guarantee geodesic integrability. By contrast, in Kerr spacetime, the isometry group alone does not guarantee geodesic integrability. However, geodesics are in fact integrable, which reflects "hidden symmetry." Namely, geodesic integrability is implied by the existence of Carter's constant [10], which follows from existence of a nontrivial Killing tensor field [11]. This shows that particle motion on a given spacetime can reveal its "hidden symmetry." In a similar sense, string motion may reveal a different type of hidden symmetry. We introduce the concept of C1-string integrability of a spacetime, which means that all allowed C1 strings are (Liouville) integrable. This is of course a necessary condition for integrability of all classical (bosonic) strings.

Recently, dynamics of classical string was widely studied in the context of AdS/CFT correspondence. While string motion is integrable in $A d S_{5} \times S^{5}$ [12] chaotic string dynamics is reported in AdS soliton [13] and in $A d S_{5} \times T^{1,1}$ [14]. The obtained chaotic solutions are also $\mathrm{C} 1$ strings. We will apply our method to those spacetimes and examine C1-string integrability. We will see that the chaotic behavior in $\mathrm{AdS}_{5} \times T^{1,1}$ is naturally understood as absence of necessary number of conserved quantity for C1-string integrability. We will also see that two geodesically integrable spacetimes which have the same isometry group can be distinguished by C1-string integrability.

We will present our method in such a way that one can fully exploit the symmetry of the spacetime.

Cohomogeneity-one strings. - Let $(M, g)$ be a $d$-dimensional spacetime. Let $G$ be the isometry group of $(M, g)$ and $\mathfrak{g}$ be its Lie algebra. An element $\xi$ of $\mathfrak{g}$ is called a Killing vector field on $(M, g)$ and generates a one-parameter family of isometries. The string that shares a such a one-parameter family with the spacetime $(M, g)$ is called a cohomogeneity-one (C1) string. More precisely, a $\mathrm{C} 1$ string has a world sheet which is foliated by one-dimensional orbits of some Killing vector field $\xi \in \mathfrak{g}$. We will call $\xi$ the homogeneity vector field.

Once the spacetime $(M, g)$ is given, one can study all $\mathrm{C} 1$ strings by considering all homogeneity vector $\xi \in \mathfrak{g}$. However, two string solutions, i.e. the world sheets $\mathcal{S}$ and $\mathcal{S}^{\prime}$, are physically the same if the world sheet $\mathcal{S}$ is sent to $\mathcal{S}^{\prime}$ by an isometry $\phi \in G$, i.e., $\mathcal{S}^{\prime}=\phi(\mathcal{S})$. We therefore classify the C1 strings by this equivalence class. In terms of homogeneity vector fields, $\xi$ and $\xi^{\prime}$ are equivalent if there exists $\phi \in G$ such that $\xi^{\prime}$ is proportional to $\phi_{*} \xi$. In a more algebraic terms, each physically different string corresponds to an element of $\mathfrak{g}$ up to equivalence by $\operatorname{Ad}_{G}$ and up to scalar multiplication, where $\operatorname{Ad}_{G}$ is the conjugation by $G$. The classification has been carried out for four-dimensional Minkowski spacetime [7] and five-dimensional anti-de Sitter spacetime [8].

Dynamics. - We consider a string that is governed by the Nambu-Goto action

$$
S=\frac{1}{2 \pi \alpha^{\prime}} \int d^{2} \sigma \sqrt{g_{a b} \frac{\partial x^{a}}{\partial \sigma^{\alpha}} \frac{\partial x^{a}}{\partial \sigma^{\beta}}},
$$

where the prefactor $\alpha^{\prime}$ is the string tension. If the string is C1, one can carry out the integral of (11) in the direction of $\xi$. This yields

$$
S=\frac{l}{2 \pi \alpha^{\prime}} \int d \lambda \sqrt{f h_{a b} \frac{d x^{a}}{d \lambda} \frac{d x^{a}}{d \lambda}}
$$

where $h_{a b}:=g_{a b}-\xi_{a} \xi_{b} / f$, with $f:=g_{a b} \xi^{a} \xi^{b}$, is the projection metric normal to $\xi$ and $l$ is a constant with a dimension of length. We define the orbit space $O$ as the quotient of $M$ by the orbits of $\xi$. Now, one observes that the action (2) is nothing but the action for a free particle in the spacetime $(O, f h)$. Thus, the original problem of finding world sheets of a $\mathrm{C} 1$ string has been reduced to that of finding geodesics on the orbit space $O$ with the weighted metric fh. In the sequel, it is beneficial to use the Polyakov-type action,

$$
S=\frac{l}{4 \pi \alpha^{\prime}} \int d \lambda\left(\frac{1}{N} f h_{a b} \frac{d x^{b}}{d \lambda} \frac{d x^{a}}{d \lambda}+N\right),
$$


TABLE I. Integrability of $\mathrm{C} 1$ strings on $\mathrm{AdS}_{5}$. Any homogeneity vector $\xi$ is equivalent to one of ten types [8]. For each type, there is a three-dimensional Abelian Lie subalgebra $\mathcal{C}$ containing $\xi$, which is shown by its generators $\langle\bullet, \bullet, \bullet\rangle$. Each type has a hidden conserved quantity $Q:=K^{a b} P_{a} P_{b}+K_{0}$. The set of five mutually commuting conserved quantities, $\left(g^{a b} P_{a} P_{b}, \xi^{a} P_{a}, X_{(1)}^{a} P_{a}, X_{(2)}^{a} P_{a}, Q\right)$, implies integrability of each C1 string. Notation: $\left(x^{i}\right)=(s, t, x, y, z, w)$ and $L, J_{x y}$, $\widetilde{K}_{z}, K_{w}$ are generators of the st rotation, the $x y$ rotation, the $s z$ boost, the $t w$ boost, etc. The product $A B$ denotes the symmetric tensor product, $(A B)^{a b}:=\left(A^{a} B^{b}+B^{a} A^{b}\right) / 2$.

\begin{tabular}{|c|c|c|c|}
\hline type & $\xi$ and Abelian subalgebra $\mathcal{C} \ni \xi$ & $K^{a b}$ & $K_{0}$ \\
\hline$(4 \mid 0)$ & $\begin{array}{l}\widetilde{K}_{x}+J_{y z}+\widetilde{K}_{z}+L ; \\
\left\langle\widetilde{K}_{x}+J_{y z}+\widetilde{K}_{z}+L, J_{x y}+J_{y z}-K_{y}+\widetilde{K}_{z},\right. \\
\left.J_{x w}-K_{w}\right\rangle \\
\end{array}$ & $\begin{array}{l}2\left(J_{x z}-K_{z}\right)\left(K_{x}+\widetilde{K}_{y}\right)+2 J_{z w}\left(J_{x w}-K_{w}\right) \\
+2\left(J_{x y}-K_{y}\right)^{2}+\left(J_{x z}-K_{z}\right)^{2}+\left(J_{y z}+\widetilde{K}_{z}\right)^{2} \\
-\left(J_{y w}+\widetilde{K}_{w}\right)^{2}-\left(J_{x y}-\widetilde{K}_{x}\right)^{2}+\left(K_{y}+L\right)^{2} \\
\end{array}$ & $(t-x)^{2}$ \\
\hline$\pm(3,1 \mid 0)$ & $\begin{array}{l}K_{x}+\widetilde{K}_{y}+J_{y z} \pm J_{x w}+a\left(J_{x y}-L \mp J_{z w}\right) \\
\left\langle K_{x}+\widetilde{K}_{y}+J_{y z} \pm J_{x w}\right. \\
\left.J_{x y}-L \mp J_{z w}, \mp J_{z w}-K_{z} \pm \widetilde{K}_{w}+L\right\rangle\end{array}$ & $\begin{array}{c}\left(J_{x z}+\widetilde{K}_{x} \pm J_{y w}+K_{y}\right)^{2}+\left( \pm J_{x w}+K_{x}-J_{y z}-\widetilde{K}_{y}\right)^{2} \\
+4 a\left[\left(J_{x z}+\widetilde{K}_{x} \pm J_{y w}+K_{y}\right)\left(\widetilde{K}_{z} \mp K_{w}\right)\right. \\
\left.+\left( \pm J_{x w}+K_{x}-J_{y z}-\widetilde{K}_{y}\right)\left(K_{z} \pm \widetilde{K}_{w}\right)\right]\end{array}$ & $\begin{array}{l}-4 a^{2} \\
\times\left[(z-s)^{2}+(w \mp t)^{2}\right]\end{array}$ \\
\hline$(2,2 \mid 0)$ & $\begin{array}{l}K_{x}+L+a J_{y z} \\
\left\langle K_{x}+L, J_{y z}, J_{x w}+\widetilde{K}_{w}\right\rangle\end{array}$ & $\begin{array}{l}\left(J_{x y}+\widetilde{K}_{y}\right)^{2}+\left(J_{x z}+\widetilde{K}_{z}\right)^{2} \\
+a^{2}\left(J_{x w}^{2}-\widetilde{K}_{x}^{2}-K_{x}^{2}-\widetilde{K}_{w}^{2}-K_{w}^{2}+L^{2}\right)\end{array}$ & $a^{2}(x+s)^{2}$ \\
\hline$(2,-2 \mid 0)$ & $\begin{array}{l}K_{x}+J_{x y}+a J_{z w} \\
\left\langle K_{x}+J_{x y}, J_{z w}, \widetilde{K}_{y}+L\right\rangle\end{array}$ & $\begin{array}{l}\left(J_{y z}-K_{z}\right)^{2}-\left(J_{y w}-K_{w}\right)^{2} \\
+a^{2}\left(J_{x y}^{2}-\widetilde{K}_{x}^{2}-K_{x}^{2}-\widetilde{K}_{y}^{2}-K_{y}^{2}+L^{2}\right) \\
\end{array}$ & $-a^{2}(y-t)^{2}$ \\
\hline$(0 \mid 2)$ & $\begin{array}{l}K_{x}+J_{x y}+a \widetilde{K}_{z} \\
\left\langle K_{x}+J_{x y}, \widetilde{K}_{z}, J_{y w}-K_{w}\right\rangle\end{array}$ & $\begin{array}{l}-\left(J_{y z}-K_{z}\right)^{2}+\left(\widetilde{K}_{y}+L\right)^{2} \\
+a^{2}\left(-J_{x y}^{2}-J_{x w}^{2}+K_{x}^{2}-J_{y w}^{2}+K_{y}^{2}+K_{w}^{2}\right) \\
\end{array}$ & $a^{2}(y-t)^{2}$ \\
\hline$(2,1,1 \mid 0)$ & $\begin{array}{l}K_{x}+\widetilde{K}_{y}+J_{x y}+L+a J_{z w}+b\left(J_{x y}-L\right) \\
\left\langle K_{x}+\widetilde{K}_{y}+J_{x y}+L, J_{z w}, J_{x y}-L\right\rangle\end{array}$ & $\begin{array}{l}\left(a^{2}-b^{2}\right)\left[\left(\widetilde{K}_{x}+K_{y}\right)^{2}+\left(K_{x}-\widetilde{K}_{y}\right)^{2}\right] \\
+2 b\left[\left(J_{x z}+\widetilde{K}_{z}\right)^{2}+\left(J_{y z}-K_{z}\right)^{2}\right. \\
\left.\quad+\left(J_{x w}+\widetilde{K}_{w}\right)^{2}+\left(J_{y w}-K_{w}\right)^{2}\right] \\
\end{array}$ & $\begin{array}{l}2 b\left(a^{2}-b^{2}\right) \\
\times\left[(x+s)^{2}+(y-t)^{2}\right]\end{array}$ \\
\hline$(2 \mid 1)$ & $\begin{array}{l}K_{x}+\widetilde{K}_{y}+J_{x y}+L+a J_{z w}+b\left(K_{y}+\widetilde{K}_{x}\right) \\
\left\langle K_{x}+\widetilde{K}_{y}+J_{x y}+L, J_{z w}, K_{y}+\widetilde{K}_{x}\right\rangle\end{array}$ & $\begin{array}{l}\left(a^{2}+b^{2}\right)\left[\left(J_{x y}-L\right)^{2}-\left(K_{x}-\widetilde{K}_{y}\right)^{2}\right]+4 b \\
\times\left[\left(J_{x z}+\widetilde{K}_{z}\right)\left(J_{y z}-K_{z}\right)+\left(J_{x w}+\widetilde{K}_{w}\right)\left(J_{y w}-K_{w}\right)\right]\end{array}$ & $\begin{array}{l}4 b\left(a^{2}+b^{2}\right) \\
\times(x+s)(y-t) \\
\end{array}$ \\
\hline$(1,1,1,1 \mid 0)$ & $\begin{array}{l}a L+b J_{x y}+c J_{z w} \\
\left\langle L, J_{x y}, J_{z w}\right\rangle\end{array}$ & $\begin{array}{l}\left(a^{2}-b^{2}\right)\left(J_{x z}^{2}+J_{x w}^{2}+J_{y z}^{2}+J_{y w}^{2}\right) \\
+\left(b^{2}-c^{2}\right)\left(\widetilde{K}_{x}^{2}+K_{x}^{2}+\widetilde{K}_{y}^{2}+K_{y}^{2}\right) \\
\end{array}$ & $\begin{array}{l}-\left(a^{2}-b^{2}\right)\left(b^{2}-c^{2}\right) \\
\times\left(x^{2}+y^{2}\right) \\
\end{array}$ \\
\hline$(1,1 \mid 1)$ & $\begin{array}{l}K_{x}+\widetilde{K}_{y}+b\left(L-J_{x y}\right)+c J_{z w} \\
\left\langle K_{x}+\widetilde{K}_{y}, L-J_{x y}, J_{z w}\right\rangle\end{array}$ & $\begin{array}{l}\left(b^{2}-c^{2}-1\right)\left[\left(\widetilde{K}_{x}+K_{y}\right)^{2}+\left(K_{x}-\widetilde{K}_{y}\right)^{2}\right] \\
+4 b\left(J_{x z} \widetilde{K}_{z}+J_{x w} \widetilde{K}_{w}-J_{y z} K_{z}-J_{y w} K_{w}\right)\end{array}$ & $\begin{array}{l}-4 b\left[\left(b^{2}-c^{2}-1\right)\right. \\
\left.(x s-y t)-b\left(z^{2}+w^{2}\right)\right]\end{array}$ \\
\hline$(0 \mid 1,1)$ & $\begin{array}{l}a K_{x}+b \widetilde{K}_{y}+c J_{z w} \\
\left\langle K_{x}, \widetilde{K}_{y}, J_{z w}\right\rangle\end{array}$ & $\begin{array}{l}\left(b^{2}+c^{2}\right)\left(J_{x z}^{2}+J_{x w}^{2}-K_{z}^{2}-K_{w}^{2}\right) \\
+\left(a^{2}+c^{2}\right)\left(J_{y z}^{2}+J_{y w}^{2}-\widetilde{K}_{z}^{2}-\widetilde{K}_{w}^{2}\right) \\
\end{array}$ & $\begin{array}{l}-\left(a^{2}+c^{2}\right)\left(b^{2}+c^{2}\right) \\
\times\left(z^{2}+w^{2}\right)\end{array}$ \\
\hline
\end{tabular}

which is equivalent to (2) and does not involve a square root. The equivalence can be seen by taking variations of (3) with respect to $N$ and then inserting the resulting equation back to (3). Note that the function $N(\lambda)$ appearing in the equation of motion (EOM) can be chosen arbitrarily because of the parametrization invariance of the action. We shall lift he problem on $O$ again to that on $M$ so as to use the high symmetry of $(M, g)$. Now, consider an action defined on $(M, f g)$ :

$$
S=\frac{l}{4 \pi \alpha^{\prime}} \int d \lambda\left(\frac{1}{N} f g_{a b} \frac{d x^{a}}{d \lambda} \frac{d x^{b}}{d \lambda}+N\right)
$$

Then, a solution to the EOM derived from the action (3) is a solution to the EOM derived from (4) which satisfies the condition $\xi_{a} \frac{d x^{a}}{d \lambda}=0$. Because $\xi^{a}$ is a Killing vector field on the spacetime $(M, f g), \xi_{a} \frac{d x^{a}}{d \lambda}$ is a conserved quantity. Therefore, the condition $\xi_{a} \frac{d x^{a}}{d \lambda}=0$ is consistent with the dynamics.

C1-string integrability. - Let us move to the Hamiltonian formalism, which is suitable for systematic discussions of the conserved quantities. The Hamiltonian for the action (4) is given by

$$
H=\frac{l}{4 \pi \alpha^{\prime}} N\left(\frac{1}{f} g^{a b} P_{a} P_{b}+1\right) .
$$

The geodesics on $(O, f h)$ is given by the solutions of the Hamiltonian system with the lifted Hamiltonian (5) that satisfy the condition $\xi^{a} P_{a}=0$.

The Hamiltonian system (5) with $2 d$-dimensional phase space is said to be Liouville integrable if there exist $d$ independent conserved quantities $Q_{i}, 0 \leqslant i \leqslant d$, such that they Poisson-commute with each other. If the system is 
Liouville integrable, the equation of motion can be solved by quadrature. We will say that the spacetime $(M, g)$ is C1-string integrable if all $\mathrm{C} 1$ strings on $(M, g)$ are Liouville integrable.

Let us recast the geodesic Hamiltonian (5) into the "potential form," by choosing $N \equiv f$ :

$$
\widetilde{H}:=\frac{l}{4 \pi \alpha^{\prime}}\left(g^{a b} P_{a} P_{b}+f\right), \quad \xi^{a} P_{a}=0 .
$$

The advantage of the potential form is that the coefficients in the kinetic term recover the original metric $g$. This enables one to take the full advantage of the symmetry of $(M, g)$. A conserved quantity $Q$ which is a polynomial in $P_{a}$ can be obtained by the Killing hierarchy [15], which is obtained by comparing the coefficients of each order of $P_{a}$ in the equation $\{\widetilde{H}, Q\}=0$, where $\{\bullet, \bullet\}$ denotes the Poisson bracket. The simplest of such conserved quantities is $Q:=X^{a} P_{a}$ which is first order in $P_{a}$. For such $Q$ and the Hamiltonian (6), the Killing hierarchy reads

$$
\nabla^{(a} X^{b)}=0, \quad X^{a} \nabla_{a} f=0 .
$$

The first equation implies that $X^{a}$ is a Killing vector on $(M, g)$, while the second implies that the norm of $\xi$ does not change in the direction of $X$.

Now, let us consider the case where $Q$ is a second order polynomial in $P_{a}$. One observes that the odd- and even-order equations in the Killing hierarchy do not couple because $\widetilde{H}$ in (6) has even-order terms only. Thus it is sufficient to consider $Q:=K^{a b} P_{a} P_{b}+K_{0}$. The condition $\{\widetilde{H}, Q\}=0$ is equivalent to

$$
\nabla^{(a} K^{b c)}=0, \quad \nabla^{a} K_{0}=K^{a b} \nabla_{b} f .
$$

The first equation states that $K^{a b}$ is a Killing tensor field. Given a Killing tensor $K^{a b}$, a function $K_{0}$ that satisfies the latter exists if and only if the consistency condition

$$
\nabla^{[a}\left(K^{b] c} \nabla_{c} f\right)=0
$$

is satisfied [15]. The quantities $X^{a} P_{a}$ and $Y^{a} P_{a}$ Poisson-commute if the vector fields $X$ and $Y$ commute. The quantities $X^{a} P_{a}$ and $K^{a b} P_{a} P_{b}+K_{0}$ Poisson-commute if $\mathcal{L}_{X} K^{a b}=0$ and $X^{a} \nabla_{a} K_{0}=0$.

As stated above, a C1 string is Liouville integrable if there exist $d$ mutually commuting conserved quantities for $\widetilde{H}$ in (6). The Hamiltonian $\widetilde{H}$ has at least two commuting conserved quantities, $\widetilde{H}$ itself and $\xi^{a} P_{a}$. We therefore propose the following procedure to examine the Liouville integrability of a $\mathrm{C} 1$ string.

Step1: Enumerate independent Killing vectors $X_{(i)}, 1 \leqslant i \leqslant I$, on $(M, g)$ which commute with $\xi$ and with each other. Then $\left(\widetilde{H}, \xi^{a} P_{a}, X_{(1)}^{a} P_{a}, \ldots, X_{(I)}^{a} P_{a}\right)$ is a set of conserved quantities that Poisson-commutes with each other. If $2+I \geqslant d$, the $\mathrm{C} 1$ string is Liouville integrable.

Step 2: If $2+I<d$, seek for second order conserved quantities $Q_{(j)}=K_{(j)}^{a b} P_{a} P_{b}+K_{0(j)}, 1 \leqslant j \leqslant J$, where $K_{(j)}^{a b}$ is a Killing tensor on $(M, g)$. If $K^{a b}$ satisfies the consistency condition (9), there is $K_{0}$ such that $Q$ is conserved. Check that $Q_{(j)}$ commute with each other and with the other conserved quantities. If $2+I+J \geqslant 2$, the $\mathrm{C} 1$ string is Liouville integrable.

If the spacetime $(M, g)$ is maximally symmetric, the Killing tensor $K^{a b}$ in the second step is always reducible, i.e., it is a linear combination of symmetric tensor products of Killing vectors. We remark that one can in principle continue the procedure above for higher order conserved quantities.

C1 surfaces in $S^{5}$. - The first space that we want to examine C1-string integrability is the 5-dimensional sphere $S^{5}$. The space $S^{5}$ is a hypersurface $\sum_{i=1}^{6}\left(x^{i}\right)^{2}=1$ in six-dimensional flat space with Cartesian coordinates $\left(x^{i}\right)$, $1 \leqslant i \leqslant 6$. The isometry group $G$ is $O(6)$. Let us first classify the homogeneity vectors $\xi$ up to conjugacy of $G$. In the case of $S^{5}$, there is only one type of them,

$$
\xi=a_{1} L_{12}+a_{2} L_{34}+a_{3} L_{56}
$$

where each $L_{i j}$ denotes the generator of the rotation in the $x^{i} x^{j}$ plane and $a_{1}, a_{2}$ and $a_{3}$ are real numbers. This may be most easily seen by the fact that $\mathfrak{o}(6)$ is isomorphic to $\mathfrak{s u}(4)$. Since any selfadjoint matrix can be diagonalized by a unitary matrix, $\mathfrak{s u}(4) / \mathrm{Ad}_{\mathrm{SU}(4)}$ can be identified with the set of traceless real diagonal matrices, which is a Cartan subalgebra of $\mathfrak{o}(6)$. A basis thereof correspond to $\left\{L_{12}, L_{34}, L_{56}\right\}$ appearing in (10).

From this Cartan subalgebra, one can choose a set $\left(\xi, X_{(1)}, X_{(2)}\right)$ of linearly independent, mutually commuting Killing vectors. Then we have a set $\left(\widetilde{H}, \xi^{a} P_{a}, X_{(1)}^{a} P_{a}, X_{(2)}^{a} P_{a}\right)$ of four mutually commuting conserved quantities. One more conserved quantity is necessary for integrability, so that we go into Step 2 in the procedure above. Since $S^{5}$ is 
maximally symmetric, any Killing tensor is reducible. We observe that $M_{i j}:=L_{2 i-1,2 j-1}^{2}+L_{2 i-1,2 j}^{2}+L_{2 i, 2 j-1}^{2}+L_{2 i, 2 j}^{2}$ $(1 \leqslant i<j \leqslant 3)$ commute with $L_{12} L_{34}$ and $L_{56}$. Let us therefore try the reducible Killing tensor

$$
K^{a b}=c_{12} M_{12}+c_{13} M_{13}+c_{23} M_{23},
$$

where $c_{12}, c_{13}, c_{23}$ are real numbers. The consistency condition (9) holds if the coefficients $c_{i j}$ satisfy

$$
\left(a_{1}^{2}-a_{2}^{2}\right) c_{12}+\left(a_{3}^{2}-a_{1}^{2}\right) c_{13}+\left(a_{2}^{2}-a_{3}^{2}\right) c_{23}=0 .
$$

Since such $c_{i j}$ always exist, there is a conserved quantity $Q=K^{a b} P_{a} P_{b}+K_{0}$ which commutes with the others [16]. Therefore, $S^{5}$ is C1-string integrable, namely, all $\mathrm{C} 1$ minimal surfaces can be obtained by quadrature.

C1 strings in $\mathrm{AdS}_{5}$. - The five-dimensional anti-de Sitter spacetime $\mathrm{AdS}_{5}$ is a pseudosphere $\eta_{i j} x^{i} x^{j}=-1$, where $\eta_{i j}=\operatorname{diag}[-1,-1,1,1,1,1]$, in the space $\left(x^{i}\right)_{1 \leqslant i \leqslant 6}=(s, t, x, y, z, w)$ with the flat metric $d s^{2}=\eta_{i j} d x^{i} d x^{j}$. The isometry group $G$ is $O(4,2)$. In contrast to the case of $S^{5}$, the homogeneity vector $\xi$ has a rich variety. They are classified into ten types [8] which are shown in Table [] where $J_{x^{i} x^{j}}:=x^{i} \partial_{x^{j}}-x^{j} \partial_{x^{i}}, K_{x^{i}}:=t \partial_{x^{i}}+x^{i} \partial_{t} \widetilde{K}_{x^{i}}:=s \partial_{x^{i}}+x^{i} \partial_{s}$, and $L:=s \partial_{t}-t \partial_{s}$ denote rotation, $t$-boost, $s$-boost, and $s t$-rotation, respectively. For each type of $\xi$, the situation concerning to integrability is similar to the case of $S^{5}$. We can choose a set $\left(\xi, X_{(1)}, X_{(2)}\right)$ of linearly independent, mutually commuting Killing vectors so that we have a set $\left(\widetilde{H}, \xi^{a} P_{a}, X_{(1)}^{a} P_{a}, X_{(2)}^{a} P_{a}\right)$ of four mutually commuting conserved quantities. One conserved quantity is missing for integrability, but there is a second order conserved quantity which commutes with them. We carried out Step 2 for each type of $\xi$ and found $K^{a b}$ that satisfies the consistency condition (9). Table I shows the resulting conserved quantity $Q=K^{a b} P_{a} P_{b}+K_{0}$ for each class. This proves the C1-string integrability of the spacetime $\mathrm{AdS}_{5}$.

C1 strings on $\mathrm{AdS}_{5} \times X^{5}$. - Let us consider a general product spacetime $(M, g)=\left(M_{1}, g_{1}\right) \times\left(M_{2}, g_{2}\right)=\left(M_{1} \times\right.$ $\left.M_{2}, g_{1} \oplus g_{2}\right)$

whose isometry group can be written as $G=G_{1} \times G_{2}$, where each $G_{i}$ is the isometry group of $\left(M_{i}, g_{i}\right)$. Then any C1 string on $M$ is defined by a Killing vector field $\xi=\xi_{1}+\xi_{2}$, where $\xi_{i} \in \mathfrak{g}_{i}$, where $\mathfrak{g}_{i}$ is the Lie algebra of $G_{i}$. Thus we have

$$
f=f_{1}+f_{2},
$$

where $f_{i}:=\left\|\xi_{i}\right\|_{g_{i}}^{2}$. This means that the potential in the Hamiltonian $\widetilde{H}$ given by (6) splits. Therefore, the Hamiltonian $\widetilde{H}$ admits a separation of variables, $\widetilde{H}=\widetilde{H}_{1}+\widetilde{H}_{2}$, where each $\widetilde{H}_{i}$ is a Hamiltonian of the potential form (6) on the space $\left(M_{i}, g_{i}\right)$. We conclude that a $\mathrm{C} 1$ string defined by $\xi$ is Liouville integrable if and only if the $\mathrm{C} 1$ strings defined by $\xi_{i}$ are Liouville integrable.

The spacetime with $M_{1}=\mathrm{AdS}_{5}$ and $M_{2}=X^{5}$, where $X^{5}$ is a five-dimensional Riemannian manifold, is of special interest in recent studies in string theory and gravity. The simplest such case is $X^{5}=S^{5}$, where $G_{1}=\mathrm{O}(4,2)$ and $G_{2}=\mathrm{O}(6)$. Because both $\mathrm{AdS}_{5}$ and $S^{5}$ are C1-string integrable, the argument above immediately implies that $\mathrm{AdS}_{5} \times S^{5}$ are C1-string integrable.

Another important case is $X^{5}=T^{p, q}$. The metric on $T^{p, q}$ is given by

$$
\begin{aligned}
g= & \sum_{i=1,2} \alpha_{i}^{2}\left(d \theta_{i}^{2}+\sin ^{2} \theta_{i} d \phi_{i}^{2}\right) \\
& +\beta^{2}\left(d \psi+q_{1} \cos \theta_{1} d \phi_{1}+q_{2} \cos \theta_{2} d \phi_{2}\right)^{2},
\end{aligned}
$$

where $q_{1}:=p, q_{2}:=q$. By the symmetry of exchanging the indices $i=1$ and $i=2$, we assume $p \leqslant q$ without loss of generality.

The group $G_{2}$ is seven-dimensional and given by $G_{2}=\mathrm{SO}(3) \times \mathrm{SO}(3) \times \mathrm{U}(1)$ up to discrete isometry whose generators are

$$
\begin{aligned}
& L_{x}^{(i)}:=\sin \phi_{i} \partial_{\theta_{i}}+\frac{\cos \phi_{i}}{\tan \theta_{i}}\left(\partial_{\phi_{i}}-\frac{q_{i} \partial_{\psi}}{\cos \theta_{i}}\right), \\
& L_{y}^{(i)}:=\cos \phi_{i} \partial_{\theta_{i}}-\frac{\sin \phi_{i}}{\tan \theta_{i}}\left(\partial_{\phi_{i}}-\frac{q_{i} \partial_{\psi}}{\cos \theta_{i}}\right), \\
& L_{z}^{(i)}:=\partial_{\phi_{i}}, \\
& L_{\psi}:=\partial_{\psi},
\end{aligned}
$$

where $i=1,2$, except for a special case in $T^{0,1}$ in which case the isometry group becomes larger [17]. Any homogeneity vector can be written as

$$
\xi=a_{1} L_{z}^{(1)}+a_{2} L_{z}^{(2)}+b L_{\psi}
$$


up to conjugacy of $G_{2}$.

Before analyzing the $\mathrm{C} 1$ strings on $\mathrm{AdS}_{5} \times T^{p, q}$, we recall that motion of a free particle on the spacetime is integrable. First, the spacetime $\mathrm{AdS}_{5}$ is geodesically integrable. Second, the space $T^{p, q}$ has three commuting Killing vectors $L_{z}^{(1)}$, $L_{z}^{(2)}$, and $L_{\psi}$. The Casimir operator of the first factor $\mathrm{SO}(3)$ defines a reducible Killing tensor $K^{a b}$ which commutes with all of them. Then the quintet $\left(g^{a b} P_{a} P_{b}, L_{z}^{(1) a} P_{a}, L_{z}^{(2) a} P_{a}, L_{\psi}^{a} P_{a}, K^{a b} P_{a} P_{b}\right)$ consists of mutually commuting, independent conserved quantities. Thus $T^{p, q}$ is geodesically integrable. Therefore, spacetimes $\operatorname{AdS}_{5} \times T^{p, q}$ are also geodesically integrable. By contrast, the behavior of the strings differs. There is a $\mathrm{C} 1$ string that is not integrable on $\mathrm{AdS}_{5} \times T^{1,1}[?]$. We examine C1-string integrability of each $\mathrm{AdS}_{5} \times T^{p, q}$ and see that C1-string integrability depends on $(p, q)$.

The Hamiltonian $\widetilde{H}$ on $T^{p, q}$ has the potential

$$
\begin{aligned}
f= & \sum_{i=1,2}\left(a_{i}^{2}\left(\alpha_{i}^{2} \sin ^{2} \theta_{i}+q_{i}^{2} \beta^{2} \cos ^{2} \theta_{i}\right)+2 q_{i} a_{i} b \beta^{2} \cos \theta_{i}\right) \\
& +b^{2} \beta^{2}+2 a_{1} a_{2} q_{1} q_{2} \beta^{2} \cos \theta_{1} \cos \theta_{2} .
\end{aligned}
$$

The Killing vectors $L_{z}^{(1)}, L_{z}^{(2)}$ and $L_{\psi}$ appearing in (19) are mutually commuting so that we can construct, by their linear combinations, a set $\left(\xi, X_{(1)}, X_{(2)}\right)$ of three linearly independent, mutually commuting Killing vectors. Thus we have a set of four mutually commuting conserved quantities $\left(\widetilde{H}, \xi^{a} P_{a}, X_{(1)}^{a} P_{a}, X_{(2)}^{a} P_{a}\right)$. We seek for one more conserved quantity, which is second order in $P_{a}$ (Step 2). There is no irreducible Killing tensor on $T^{p, q}$ which commutes with $\xi$. Reducible Killing tensors which commute with $\xi$ are

$$
\begin{aligned}
K_{(i)} & =L_{x}^{(i) 2}+L_{y}^{(i) 2}+L_{z}^{(i) 2}-q_{i}^{2} L_{\psi}^{2} \\
& =\partial_{\theta_{i}}^{2}+\frac{1}{\sin ^{2} \theta_{i}}\left(\partial_{\phi_{i}}-q_{i} \cos \theta_{i} \partial_{\psi}\right)^{2}, \quad i=1,2 .
\end{aligned}
$$

They are related to the (inverse) metric by $g^{a b}=K_{(1)}^{a b} / \alpha_{1}^{2}+K_{(2)}^{a b} / \alpha_{2}^{2}+L_{\psi}^{a} L_{\psi}^{b} / \beta^{2}$. Thus $K_{(1)}$ and $K_{(2)}$ are not independent and it is enough to examine if there is a conserved quantity $Q=K_{(1)}^{a b} P_{a} P_{b}+K_{0}$ (rather than to consider the Killing hierarchy for all combinations of $K_{(1)}$ and $\left.K_{(2)}\right)$. We observe that the potential $f$ becomes separable, $f=f_{(1)}\left(\theta_{1}\right)+f_{(2)}\left(\theta_{2}\right)$, for any homogeneity vector $\xi$, if the last term vanishes in the expression (20). In that case, it follows immediately that $K_{0}=\left(\alpha_{1}^{2} / a_{1}^{2}\right) f_{(1)}$ is a solution to the latter equation in the Killing hierarchy (8) with $K^{a b}=K_{(1)}^{a b}$. Thus the quintet $\left(\widetilde{H}, \xi^{a} P_{a}, X_{(1)}^{a} P_{a}, X_{(2)}^{a} P_{a}, Q\right)$ is a set of mutually commuting conserved quantities and proves the integrability of the $\mathrm{C} 1$ string defined by $\xi$. On the other hand, if the last term in the expression (20) does not vanish, the $\mathrm{C} 1$ string defined by $\xi$ is not integrable. The potential $f$ including the term which depends on both $\theta_{1}$ and $\theta_{2}$ fails to satisfy the consistency condition (9) with $K^{a b}=K_{(1)}^{a b}$ [18]. To summarize, the C1 string defined by $\xi$ is integrable if and only if the last term in (20) is identically zero.

This leads to the following results. First, the space $T^{0, q}$ is C1-string integrable. Thus, by the argument above on direct products, $\mathrm{AdS}_{5} \times T^{0, q}$ is C1-string integrable. Second, the space $T^{p, q}$ with $p q \neq 0$ is not C1-string integrable (as far as conserved quantities in order two or less in $P_{a}$ are concerned). Namely, the C1 strings defined by $\xi=a_{1} L_{z}^{(1)}+b L_{\psi}$ and by $\xi=a_{2} L_{z}^{(2)}+b L_{\psi}$ are integrable but the other C1 strings are not integrable. Thus, $\operatorname{AdS}_{5} \times T^{p, q}(p q \neq 0)$ is not C1-string integrable. We remark that the metric (14) on $T^{p, q}$ satisfies the property called Sasaki-Einstein and $\mathrm{AdS}_{5} \times T^{p, q}$ partially retains supersymmetry when $p=q=1, \alpha_{1}^{2}=\alpha_{2}^{2}=1 / 6$, and $\beta^{2}=1 / 9$. C1 integrability does not depend on whether the spacetime retains supersymmetry or not.

Conclusion. - We presented a method with which one can systematically analyze conserved quantities and integrability of strings of class $\mathrm{C} 1$ (cohomogeneity-one). We applied the method to several important spaces and spacetimes, $S^{5}, \mathrm{AdS}_{5}, \mathrm{AdS}_{5} \times S^{5}$, and $\mathrm{AdS}_{5} \times T^{p, q}$. We found that the first three space and spacetimes are C1-string integrable, in which all $\mathrm{C} 1$ strings are Liouville integrable. On the other hand, C1-string integrability of $\mathrm{AdS}_{5} \times T^{p, q}$, depends on $(p, q)$. Namely, $T^{0, q}$ are C1-string integrable while $T^{p, q}$ with $p q \neq 0$ are not. Thus the chaotic behavior in $\operatorname{AdS}_{5} \times T^{1,1}$ is naturally understood as absence of necessary conserved quantity for C1-string nonintegrability.

As in the example of Kerr spacetime, geodesic integrability can probe hidden symmetry which is not implied by the isometry group. C1-string integrability discriminates $\mathrm{AdS}_{5} \times T^{p, q}$, which have the same isometry group and are geodesically integrable. Therefore, C1-string integrability (or nonintegrability) probes finer hidden symmetry. Intuitively, by the C1-string integrability, one is measuring the remaining symmetry after a "piece" of symmetry (corresponding to the homogeneity vector) is removed. One can generalize the C1-string integrability to higherdimensional objects, C1-membrane integrability and so forth, which might bring further useful information of the spacetime. 
The authors thank Y. Yasui and K. Yoshida for useful discussions. This work was supported by JSPS KAKENHI Grant Number JP16K05358 (HI).

[1] A. Vilenkin and E. P. S. Shellard, Cosmic Strings and Other Topological Defects (Cambridge Univ. Press, Cambridge, 1994).

[2] C. J. Burden and L. J. Tassie, Aust. J. Phys., 35, 223 (1982); ibid. 37, 1 (1984);

[3] V. P. Frolov, V. Skarzhinsky, A. Zelnikov and O. Heinrich, Phys. Lett. B 224, 255 (1989); V. P. Frolov, S. Hendy and J. P. De Villiers, Class. Quant. Grav. 14, 1099 (1997);

[4] H. J. de Vega, A.L. Larsen, and N. Sanchez, Nucl. Phys. B427, 643 (1994); A. L. Larsen and N. Sanchez, Phys. Rev. D 50, 7493 (1994); ibid. 51, 6929 (1995); H. J. de Vega and I. L. Egusquiza, Phys. Rev. D 54, 7513 (1996).

[5] K. Ogawa, H. Ishihara, H. Kozaki, H. Nakano and S. Saito, Phys. Rev. D 78, 023525 (2008); K. Ogawa, H. Ishihara, H. Kozaki and H. Nakano, ibid. 79, 063501 (2009).

[6] T. Igata and H. Ishihara, Phys. Rev. D 81, 044024 (2010); ibid. 82, 044014 (2010). T. Igata, H. Ishihara and K. Nishiwaki, Phys. Rev. D 86, 104020 (2012).

[7] H. Ishihara and H. Kozaki, Phys. Rev. D 72, 061701(R) (2005).

[8] T. Koike, H. Kozaki and H. Ishihara, Phys. Rev. D 77, 125003 (2008).

[9] H. Kozaki, T. Koike and H. Ishihara, Class. Quant. Grav. 27, 105006 (2010).

[10] B. Carter, Phys. Rev., 174, 1559 (1968).

[11] M. Walker and R. Penrose, Commun. Math. Phys. 18, 265-274 (1970).

[12] G. Mandal, N. V. Suryanarayana and S. R. Wadia, Phys. Lett. B 543, 81 (2002).

I. Bena, J. Polchinski and R. Roiban, Phys. Rev. D 69, 046002 (2004).

[13] P. Basu, D. Das and A. Ghosh, Phys. Lett. B 699, 388 (2011); T. Ishii, K. Murata and K. Yoshida, Phys. Rev. D 95, no. 6, 066019 (2017).

[14] P. Basu and L. A. Pando Zayas, Phys. Lett. B 700, 243 (2011); Phys. Rev. D 84, 046006 (2011). Y. Asano, D. Kawai, H. Kyono and K. Yoshida, JHEP 1508, 060 (2015).

[15] T. Igata, T. Koike, and H. Ishihara, Phys. Rev. D 83, 065027 (2011).

[16] An example is $c_{12}=\left(a_{1}^{2}-a_{3}^{2}\right), c_{13}=\left(a_{1}^{2}-a_{2}^{2}\right), c_{23}=0$ which leads to $K_{0}=\left(a_{1}^{2}-a_{2}^{2}\right)\left(a_{1}^{2}-a_{3}^{2}\right)\left(x_{1}^{2}+x_{2}^{2}\right)$ hence to the corresponding $Q$. Other choices of $c_{i j}$ give conserved quantities which are constructed by $Q$ and $\left(\tilde{H}, \xi^{a} P_{a}, X_{(1)}^{a} P_{a}, X_{(2)}^{a} P_{a}\right)$

[17] In the case $T^{0,1}=S^{2} \times S^{3}$ (topologically) and $\alpha_{2}=\beta$, the symmetry enhances to $G_{2}=\mathrm{SO}(3) \times \mathrm{SO}(4)$. Although there might be in principle a homogeneity vector $\xi$ different from (19), actually $\xi$ in this case is always given by (19) up to conjugacy of the enhanced $G_{2}$.

[18] Eq. (9) states that $\omega_{b}:=K_{b}{ }^{c} \nabla_{c} f$ is a closed 1-form. However, in coordinates $\left(\theta_{1}, \phi_{1}, \theta_{2}, \phi_{2}, \psi\right)$, one has $\omega_{\theta_{2}}=0$ while $\omega_{\theta_{1}}$ depends on $\theta_{2}$. Thus $\omega_{b}$ cannot be closed. 